\title{
Design of Cylindrical Shells using the Single Perturbation Load Approach - Potentials and Application Limits
}

\author{
Benedikt Kriegesmann ${ }^{1}$, Eelco L. Jansen ${ }^{2}$, Raimund Rolfes ${ }^{2}$ \\ ${ }^{1}$ Hamburg University of Technology, \\ Am Schwarzenberg-Campus 4,21073 Hamburg, Germany \\ ${ }^{2}$ Institute of Structural Analysis, Leibniz Universität Hannover, \\ Appelstrasse 9A, 30167 Hannover, Germany
}

\begin{abstract}
The Single Perturbation Load Approach (SPLA) is a promising deterministic procedure on the basis of mechanical considerations to determine reasonable design loads for cylindrical shells in axial compression. In this paper, two main issues are identified that should be understood better in order to appreciate the potential and the limits of application of the SPLA. Firstly, the question whether a single perturbation load in general represents a "worst case" imperfection is addressed. Secondly, the influence of the stiffness properties of the shell on the quality of the SPLA predictions is studied. Finally, an indicator is suggested which identifies, based on the cylinder stiffness properties, whether the SPLA is conservative for a considered shell or not.
\end{abstract}

Key words: Buckling, Design, Shell structures, single perturbation load approach

\section{Introduction}

Cylindrical shells under axial compression are prone to buckling, where the experimental buckling load is usually significantly smaller than the theoretical buckling load. Koiter [1] found geometric imperfections to be a main reason for this gap, while also boundary imperfections can have a significant influence on the buckling load [2]. Weingarten et al. [3] proposed a lower bound of the buckling load, based on experimental results available at that time. This lower bound has been adapted in the knockdown factors given by NASA SP-8007 [4]. This design rule turned out to be overly conservative for modern shells [5]-[8] and has been developed for metallic shells only. Composite cylindrical shells can show very different sensitivities depending on the laminate setup [7], [8], which is not captured by NASA SP-8007. The guideline is reworked in the framework of the currently running Shell Buckling Knockdown Factor project [9].

In order to overcome the limitations of the lower bound design philosophy, different approaches have been followed over the years. One group of approaches are probabilistic design procedures (see, e.g., [6], [8], [10]-[12]). Since the imperfections are of random nature, the idea of these approaches is to capture this randomness and to determine the stochastic distribution of the buckling load. Based on the stochastic distribution and a chosen level of reliability, a lower bound is obtained. The problem of this type of approach is that it requires imperfection measurements, which are often not available, especially in an early design phase. 
It is noted that nondestructive experimental approaches - applicable in structural development stages in which specimens are already available - have recently been presented [13], [14], in the line of the vibration correlation technique originally proposed by Singer [15], [16].

A deterministic design concept to account for imperfection sensitivity in an early design phase is the Single Perturbation Load Approach (SPLA), which has been proposed by Hühne et al. [7]. The idea is to apply a lateral perturbation load $P$ when determining the buckling load of a cylindrical shell. For increasing perturbation loads, the bucking load decreases, until the perturbation load reaches a certain level $P_{1}$. The associated buckling load $N_{1}$ has been defined as design load by Hühne. This approach is currently further developed in the context of the European research project DESICOS (New Robust DESign Guideline for Imperfection Sensitive COmposite Launcher Structures) [17].

One major restriction regarding the applicability of the SPLA has been raised by Friedrich and Schröder [18]. They showed that the SPLA can be applied for displacement controlled simulations and experiments, but that for load driven scenarios the applicability of the approach has restrictions. This paper focuses on displacement controlled simulations and tests, covering the majority of published experiments. However, in the present contribution, two further issues are identified that should be understood better in order to appreciate the potential and the limits of application of the SPLA. Firstly, the question whether a single perturbation load in general represents a "worst case" imperfection will be addressed. Secondly, the influence of the stiffness properties of the shell on the SPLA predictions will be studied in detail.

With regard to the second issue, a key observation is that one of the composite cylindrical shells Hühne tested showed a lower buckling load in experimental test than the design load $N_{1}$ according to the SPLA. Furthermore, probabilistic analyses of the same type of shells showed that also for a second cylinder the design load $N_{1}$ has an unacceptably small reliability [8]. Probabilistic analyses in which only geometric imperfections are taken into account indicated that the SPLA covers the effect of geometric imperfection, but not the effect of other types of imperfection such as boundary imperfections [19]. For shells which are sensitive to geometric imperfections, the SPLA nevertheless provides a conservative lower bound. Arbelo et al. [20] applied the SPLA to a large number of cylinders showing further cases in which the buckling load in test was lower than $N_{1}$. What is lacking today is a criterion that indicates the range of applicability of the SPLA. This is what the current paper aims to contribute.

In order to reveal the limitations of the SPLA, different laminate configurations should be further investigated. In particular, the strikingly different behavior of two particular shells (Z07 and Z09) that have been investigated both experimentally and numerically in earlier studies, is addressed in the current paper. These composite shells have the same layers, but a reversed stacking sequence. Having the same in-plane stiffness, the only difference is the sign of specific coefficients of the bending stiffness matrix and in particular of the bending-stretching coupling stiffness matrix, which results in significantly different buckling behavior and imperfection sensitivity. The current paper focuses on the behavior of these two particular shells and aims at shedding light on their different type of behavior.

A similar pair of cylinders (Z32/Z33) has been investigated by Geier et al. [21], who explained the change of buckling load caused by reversing the stacking sequence. Castro et al. [22] deeply investigated these two cylinders in presence of a perturbation load, showing their significantly differing sensitivity. For the cylinders Z07 and Z33, which both show a high imperfection 
sensitivity, Castro et al. [23] furthermore investigated the influence of different imperfection shapes on the buckling load and lower bounds of these shells.

In the present paper, the buckling behavior and the characteristics of the imperfection sensitivity are monitored for a range of shell properties varying continuously from the Z09 properties to the Z07 properties. Numerical investigations are carried out with both, the SPLA approach and a semi-analytical implementation of Koiter's b-factor method. The single mode b-factor method gives an estimate of the imperfection sensitivity and can also provide an estimate of the decrease of the load carrying capability of the shell for a given imperfection [12]. Koiter's b-factor method will be used to assess the applicability of the SPLA by comparing the prediction of the imperfection sensitivity obtained using the b-factor method with the prediction obtained using the SPLA. With the help of the b-factor method and the analytical approach given by Geier et al. [21], the possibility is investigated to formulate a simple, practical indicator to identify cases in which the SPLA should be used with caution.

\section{The Single Perturbation Load Approach - Status}

The basic idea of the SPLA proposed by Hühne et al. [7] is given in the following section. Furthermore, the results of applying this approach to various types of shells are given and it is evaluated which other types of imperfections are captured by the SPLA.

\subsection{General Concept}

When applying a lateral perturbation load $P$ to a cylindrical shell as shown in Figure 1, left, the buckling load $N$ is reduced compared to the buckling load of perfect, unperturbated shell. However, when the perturbation load exceeds a certain value $P_{1}$, a further increase does not decrease the collapse load any further (see Figure 1, right). The associated buckling load $N_{1}$ is defined as design load.
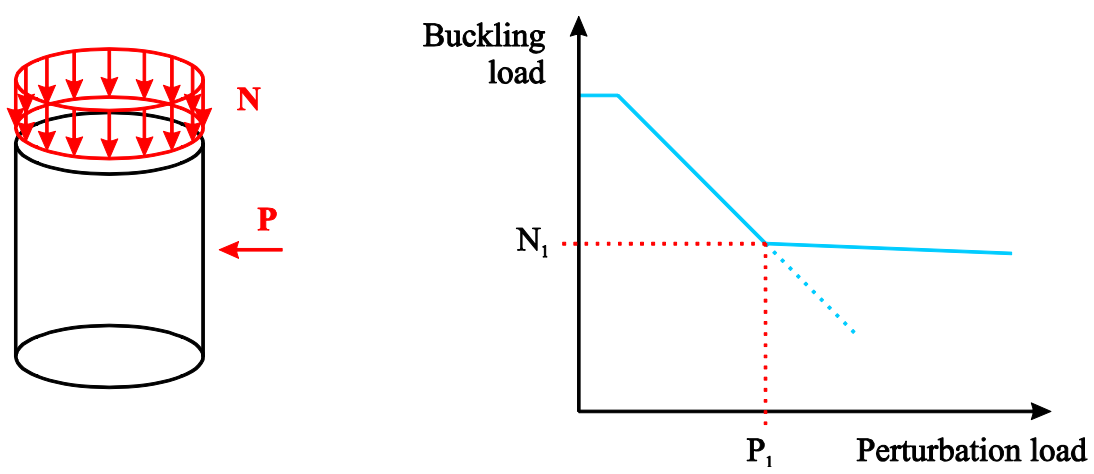

Figure 1: Basic idea of the SPLA

For very small perturbation loads, the perturbation load hardly influences the buckling load. When the perturbation load exceeds $P_{1}$, a small drop occurs in the load-displacements curve, as shown in Figure 2 (beginning with $\mathrm{P}=70 \mathrm{kN}$ ) for an aluminum cylinder tested at NASA Langley Research Center [24]. (This cylinder will be referred to as NASA test article, NTA, in the following.) This first point of instability is indicated by a blue dotted line in Figure 1, right. At this point, the cylinder buckles locally, while the cylinder is still able to carry more load until the global buckling load is reached. The mechanical mechanism is discussed in detail in [7]. 
It is difficult to predict in advance the order of magnitude of $P_{1}$. Therefore, a multitude of buckling analyses is required to determine $P_{1}$. If the drop in the load displacement curve occurs, the design load obtained can be regarded as a conservative approximation of $N_{1}$. For a faster determination of $P_{1}$, Steinmüller et al. [25] gave an empirical equation to approximate $P_{1}$ for fiber composite cylinders based on the laminate setup.

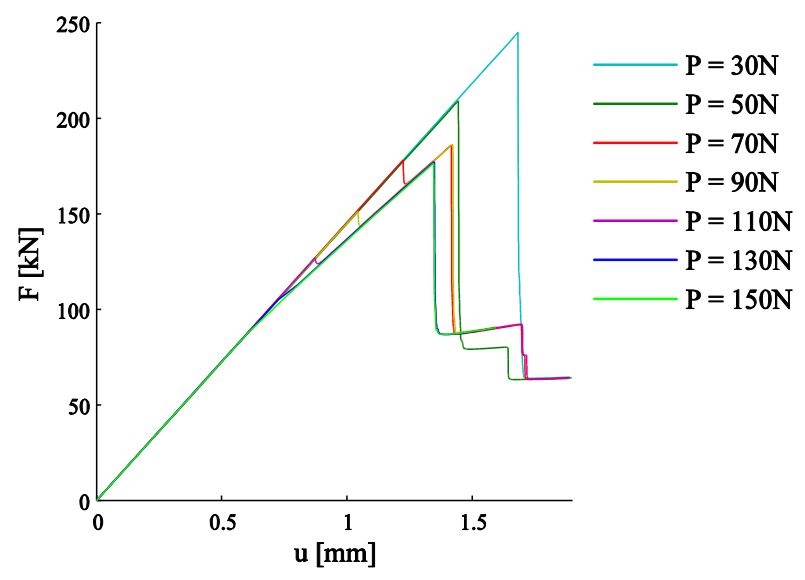

Figure 2: Load-displacement curves from simulation of the NASA test article (NTA) for different perturbation loads

\subsection{Is the Single Perturbation Load a "Worst Case" Imperfection?}

Before tackling the question for which types of shells the SPLA is conservative, it is evaluated in how far the single perturbation can be considered as a "worst case" imperfection. Therefore, subsequently two other types of perturbations or imperfections are considered in order to evaluate their influence on the buckling load compared to a single perturbation load. Furthermore, the influence of multiple perturbations is considered.

Instead of applying a single perturbation load that induces a buckle, a single dimple can be applied as geometric imperfection as shown in Figure 3. Besides that, the simulation is performed in the same way as for the SPLA, i.e. using the same boundary conditions.

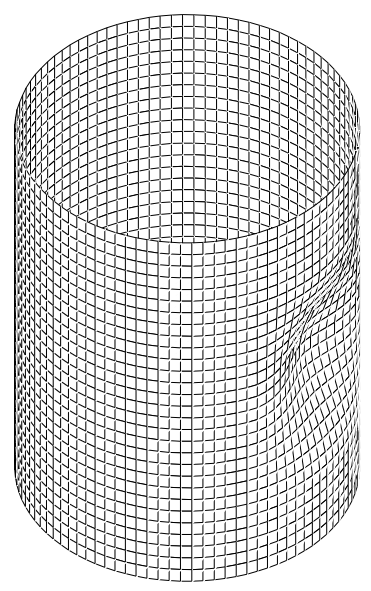

Figure 3: Cylinder model with initial single dimple imperfection 
When increasing the amplitude $a$ of this single dimple, the buckling load decreases up to a certain value (see Figure 4 and Figure 5, where the amplitude $a$ is given as fraction of the wall thickness $t$ ). In fact, the behavior looks similar to the one observed under a single perturbation load.

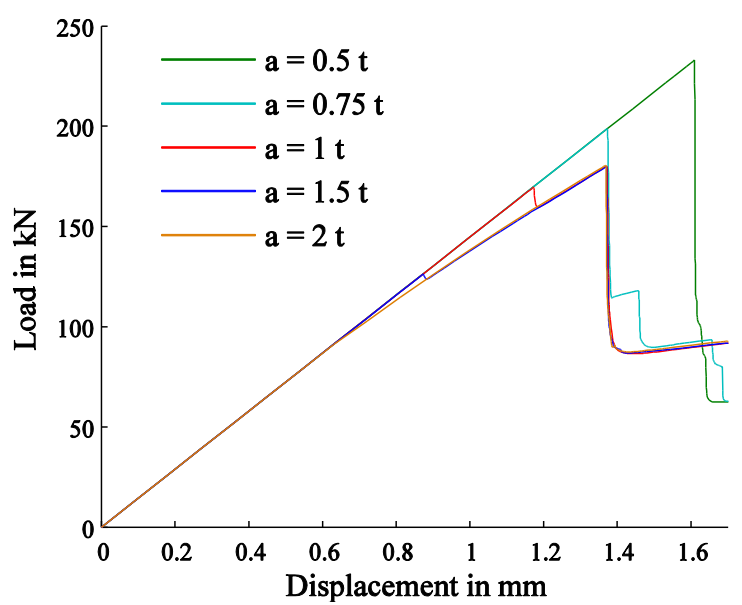

Figure 4: Load over end shortening for NTA with a single dimple imperfection

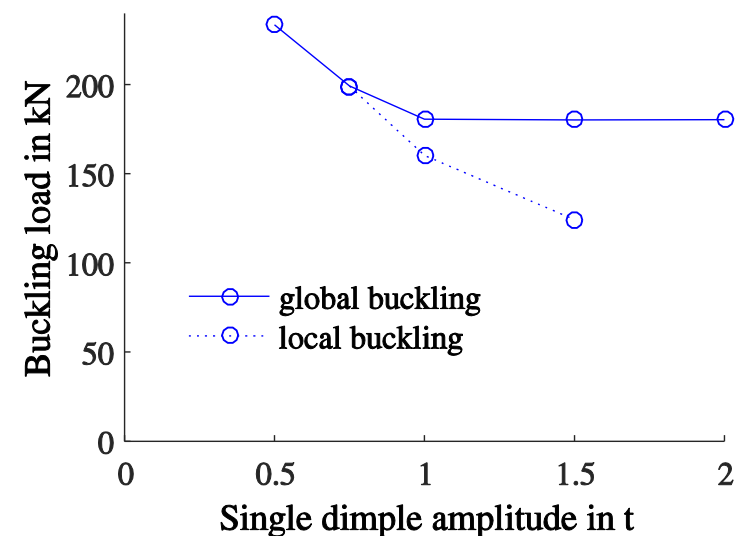

Figure 5: Buckling load over single dimple imperfection amplitude for NTA

The approach of increasing the amplitude of a single dimple imperfection (SDI) has been performed for the shells Z07, Z09 and NTA. The results shown in Table 1 indicate that the behavior in presence of a SDI yields a lower bound which is very close to the one obtained with a perturbation load. The same observation is reported in [26] and [23], also for the cylinders Z32 and Z33.

\begin{tabular}{lccc}
\hline \hline Shell & Z07 & Z09 & NTA \\
\hline$N_{1}$ (SPLA) in kN & 21.8 & 16.7 & 187 \\
$N_{1}$ (SDI) in kN & 21.6 & 16.6 & 180 \\
\hline \hline
\end{tabular}

Table 1: Lower bounds given by single buckle approach and single dimple imperfection approach

As similar effect as seen for a single perturbation load and a single dimple is observed for unreinforced circular cutouts. Already in 1968 Tennyson [27] observed in experiments that from a certain cutout size on the buckling load decreases much more moderately for an increasing cutout size. Toda [28] confirmed this finding for cylinders with two opposed circular cutouts. Arbelo et al. [29] performed numerical studies with cylinder Z33, in which a perturbation load is combined with a cutout. Keeping the perturbation load constant and varying the cutout size and vice versa both lead to a similar behavior as described for the SPLA, but the lower bound obtained by combining both effects is smaller than when only applying a perturbation load.

In order to assess the influence of a locally concentrated boundary imperfection, a small edge extension is applied to the cylinder as shown in Figure 6 for the NTA. This edge extension is compressed in a first step, before the whole shell is loaded. The edges remain clamped in the 
whole simulation. The influence of such imperfection on the buckling load is shown in Figure 7 and Figure 8 for different amplitudes $a$ and width $y_{t}$ of the boundary imperfection.

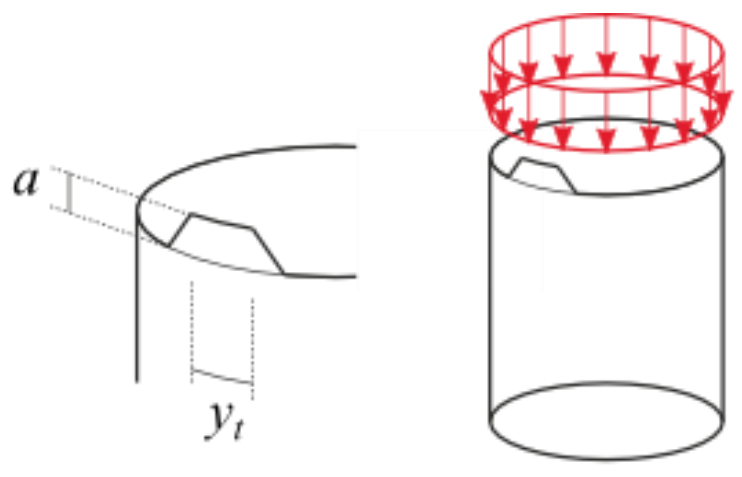

Figure 6: Cylinder model with local boundary imperfection through application of edge extension

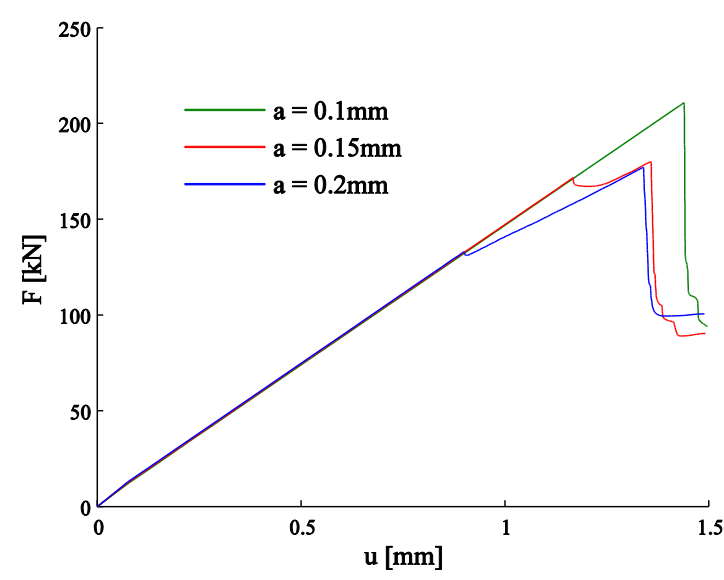

Figure 7: Load versus end shortening for three different loading imperfection amplitudes for NTA

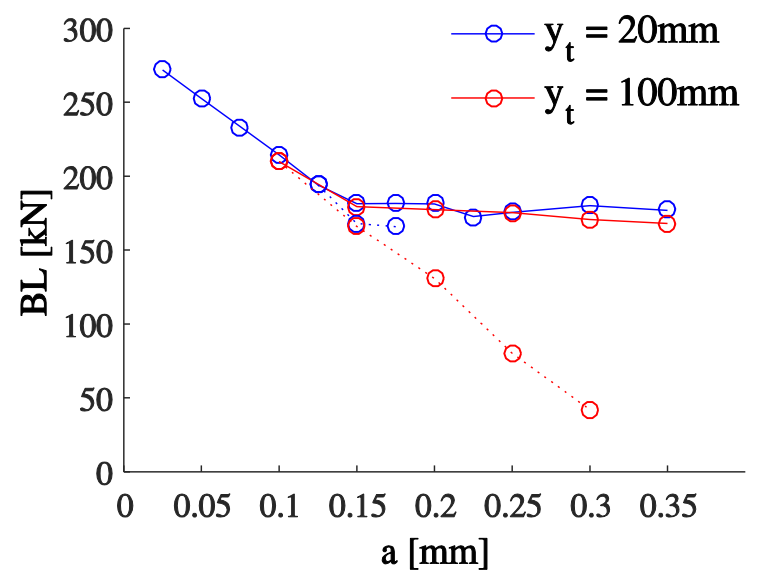

Figure 8: Buckling load versus loading imperfection amplitude for NTA

The buckling behavior in presence of a local boundary imperfection (LBI) is similar to the one in presence of a perturbation load (see Figure 7 and Figure 8). Also the lower bound obtained is almost the same (see Table 2).

\begin{tabular}{lccc}
\hline \hline Shell & Z07 & Z09 & NTA \\
\hline$N_{1}$ (SPLA) in kN & 21.8 & 16.7 & 187 \\
$N_{1}$ (LBI) in kN & 21.8 & 16.4 & 181 \\
\hline
\end{tabular}

Table 2: Lower bounds given by single buckle approach and local boundary imperfection (LBI) approach

Looking at the deformation shows that the boundary imperfection induces a single buckle which moves from the edge to the center as the load increases (for more details see [30]). 
The results shown above indicate that a single dimple induced by a single perturbation load indeed covers the effect of a local geometric imperfection, a cutout and of a local boundary imperfection. However, the behavior looks somewhat different in presence of multiple perturbations or local imperfections.

In a study performed with the NTA cylinder, the number of perturbations has been increased in FE simulations. (The distance of the perturbation loads turned out to have no influence.) Figure 9 to Figure 11 display the axial load over axial end shortening. For perturbation loads where the first point of instability (first drop in load-displacement curve) coincides with collapse, the number of perturbation loads turned out to have no impact.

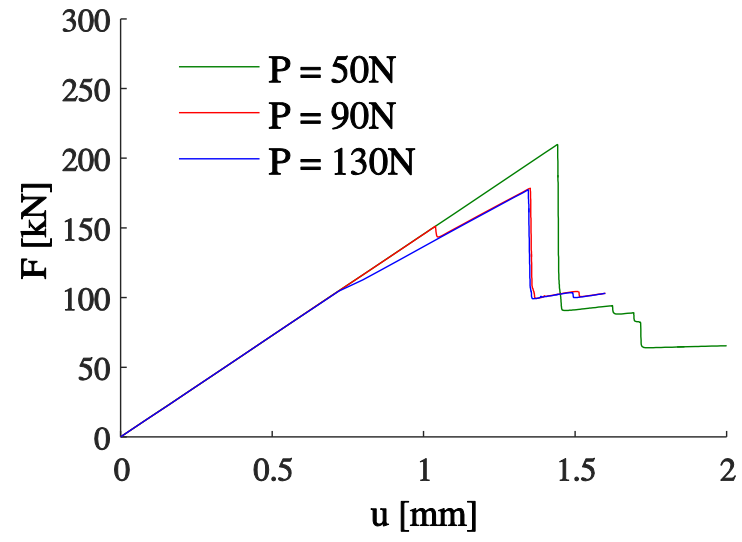

Figure 9: Load-displacement curve for NTA cylinders with one perturbation load

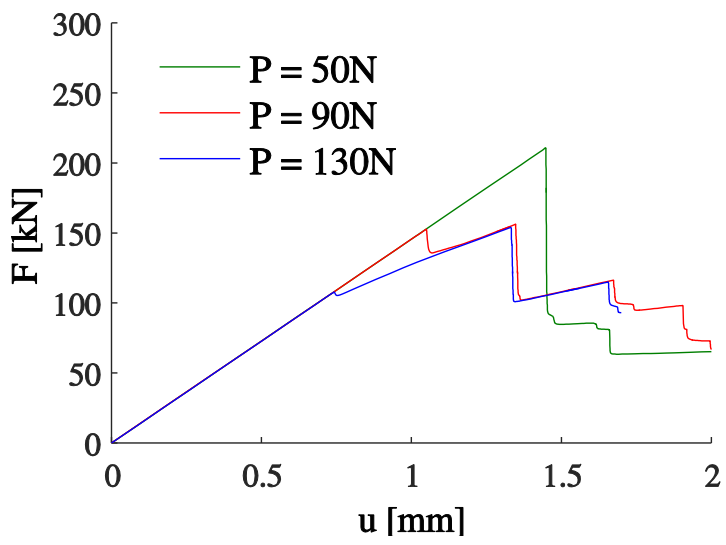

Figure 10: Load-displacement curve for NTA cylinders with two perturbation loads

In those cases, where the first point of instability does not lead to global collapse, the (later occurring) collapse load does decrease as the number of perturbation loads increases. Hence, if the SPLA is performed with multiple perturbation loads (which makes it a MPLA), the lower bound decreases as shown in Figure 12. Actually, for a certain number of perturbation loads, the cylinder smoothly enters the postbuckling region (e.g., for seven perturbation loads of $150 \mathrm{~N}$ on NTA, see Figure 11), and the perturbation load approach does not provide a lower bound as shown in Figure 1. This behavior was also observed by Arbelo et al. [31]. The same behavior is observed for instance by Castro et al. [23] when applying non-local imperfection patterns like axisymmetric imperfection or buckling modes. For an increasing amplitude, the buckling load decreases without showing a lower bound. 


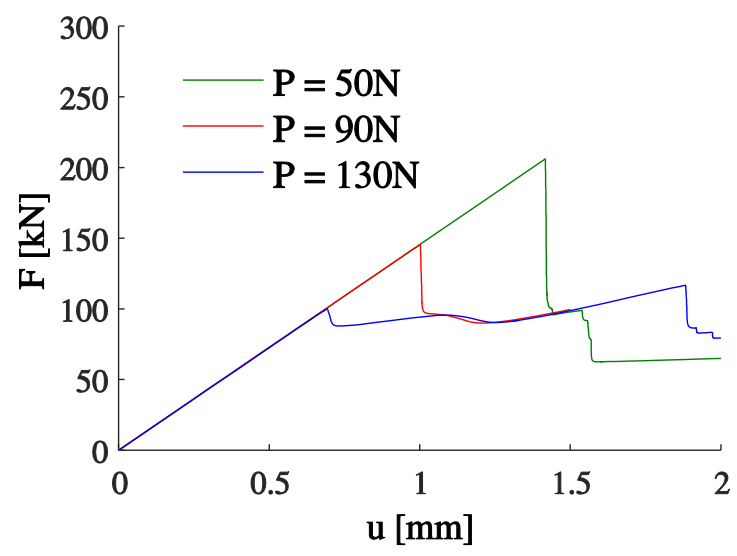

Figure 11: Load-displacement curve for NTA cylinders with seven perturbation loads

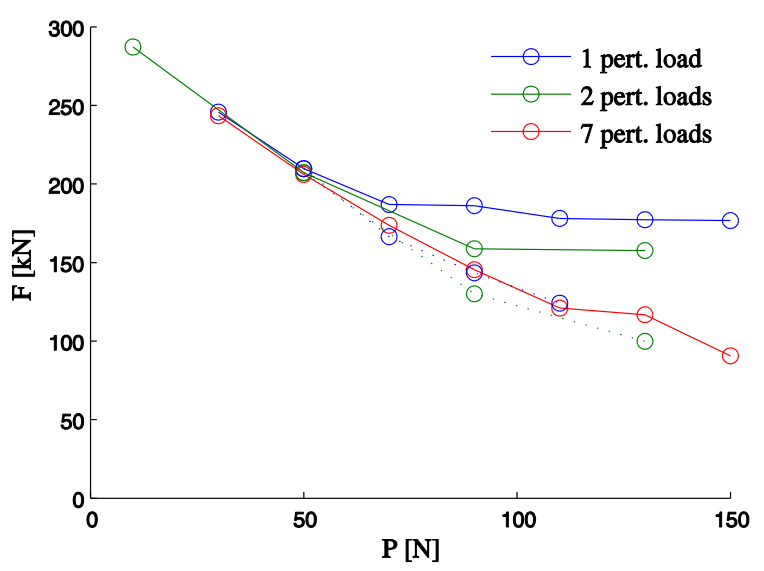

Figure 12: Buckling load over perturbation load for NTA cylinders with up to seven perturbation loads

It is concluded that the single perturbation load approach covers also other locally limited defects, but not in general any global imperfections. Furthermore, it has been shown in [19] that for some shells a slight inclination of the loading plane, causing a bending moment, can reduce the buckling load significantly more than the single dimple induced within the SPLA. Hence, there are some sources for decreasing the buckling load beyond the lower bound given by the SPLA.

Friedrich and Schröder [18] emphasize that in an explicit, load driven analysis the snap does not lead to a stable equilibrium. Hence, the lower bound (if there still exists one) is shifted down to the end of the blue dotted line in Figure 1 on the right.

\subsection{Influence of stiffness properties on SPLA predictions}

In order to evaluate the applicability of the SPLA, the lower bound given by the SPLA is compared to test results. This has already been done by Arbelo et al. [20] for a lot of cylinders given in the literature. This summary of results shall be complemented in this paper and serve as basis for further considerations.

All lay-ups given in the following are defined from inside to outside, and $0^{\circ}$ refers to the cylinder axis. Furthermore, all cylinders considered had clamped edges in tests and therefore in all numerical simulations performed clamped edges were chosen as boundary conditions.

Hühne et al. [7] tested a set of composite shells (denominated as Z07-Z12) and determined the lower bound $N_{1}$ for these shells. Degenhardt et al. [32] tested ten nominally identical shells (denominated as Z15, Z17, Z18 and Z20-Z26) with the same laminate as Z07. The results of the mentioned works are summarized in Table 3 . The slight differences between the first results of Hühne from Ref. [33] and the more recent work of Wagner and Hühne [34] do not affect the conclusions drawn in the present paper.

\begin{tabular}{lcccccc}
\hline \hline Name & Laminate & $\begin{array}{c}\text { Perf. BL } \\
\text { in kN, } \\
\text { from [33] }\end{array}$ & $\begin{array}{c}\text { Min. exp. } \\
\text { BL in kN, } \\
\text { from [33] }\end{array}$ & $\begin{array}{c}N_{1} \text { in kN, } \\
\text { from [33] }\end{array}$ & $\begin{array}{c}\text { Min } \rho_{\text {exp }} \\
\text { from [34] }\end{array}$ & $\begin{array}{c}\text { PsPLA from } \\
\text { [34] }\end{array}$ \\
\hline Z07, Z08, & {$[ \pm 24, \pm 41]$} & 31.8 & 21.3 & 17.4 & 0.64 & 0.57
\end{tabular}




\begin{tabular}{lllllll}
\hline Z15-Z26 & & & & & \\
Z09 & {$[ \pm 41, \pm 24]$} & 17.0 & 15.7 & 14.7 & 0.89 & 0.9 \\
Z10, Z11 & {$[24, \pm 41,-24]$} & 23.0 & 15.7 & 13.8 & 0.65 & 0.63 \\
Z12 & {$[ \pm 45,0,-79]$} & 22.0 & 18.6 & 20.2 & 0.79 & 0.94 \\
\hline \hline
\end{tabular}

Table 3: Buckling loads (BL) and design loads for the composite shells tested by Hühne et al [7], [33], [34]

Geier et al. [21] gave test and analysis results of two CFRP cylinders which have the same layers, but inverted stacking sequence. The nominal length, radius and ply thickness are the same as for the cylinders given in [7] $\left(L=510 \mathrm{~mm}, R=250 \mathrm{~mm}, t_{p l y}=0.125 \mathrm{~mm}\right)$. Castro et al. [22] determined the lower bound $N_{1}$ according to SPLA. The results summarized in Table 4 show that for cylinder Z33 $N_{1}$ is conservative, where for Z32 it exceeds the test result. (It should be noted that compared to [21] the stacking sequence is reversed, since in [21] the stacking was defined from outside to inside.)

\begin{tabular}{llccc}
\hline \hline Name & Laminate & $\begin{array}{c}\text { Perf. BL in kN, } \\
\text { from [21] }\end{array}$ & $\begin{array}{c}\text { Min. exp. BL in } \\
\text { kN, from [21] }\end{array}$ & $\begin{array}{c}N_{1} \text { in kN, } \\
\text { from [22]* }\end{array}$ \\
\hline Z32 & {$\left[\mp 51, \mp 45, \mp 37, \mp 19,0_{2}\right]$} & 100 & 89 & $\sim 94$ \\
Z33 & {$\left[0_{2}, \pm 19, \pm 37, \pm 45, \pm 51\right]$} & 192 & 173 & $\sim 121$ \\
\hline \hline
\end{tabular}

*in [22], the values for $N_{1}$ are not given in numbers, but are extracted from diagram

Table 4: Buckling loads (BL) and design loads for the composite shells given in [20]

Waters [35] tested a set of composite cylindrical shells at NASA Langley Research Center (LaRC), which are assigned as AW-Cyl (see also [6]). The nominal dimensions of these cylinders are $L=355.6 \mathrm{~mm}, R=203.2 \mathrm{~mm}$ and $t_{p l y}=0.127 \mathrm{~mm}$. In Table 5, the experimentally determined buckling loads (BL), the results of the perfect shell analysis as well as the design load $N_{1}$ given by the SPLA are shown. The cylinder AW-Cyl-5-1 was damaged prior to testing, but tested nevertheless. Therefore, it is not critical that the design load exceeds the experimental buckling load. In case of AW-Cyl-2-1 the design load firstly exceeded the experimental buckling load, too. However, a significant material non-linearity occurred in the prebuckling range of the shell. When using a simple elastic-plastic material model for in-plane shear, the obtained $N_{1}$ load is below the experimental result.

\begin{tabular}{llccc}
\hline \hline Name & Laminate & $\begin{array}{c}\text { Perf. BL in kN, } \\
\text { from [6] and [35] }\end{array}$ & $\begin{array}{c}\text { Experimental BL in kN, } \\
\text { from [6] and [35] }\end{array}$ & $\begin{array}{c}\text { SPLA } \\
N_{1} \text { in kN }\end{array}$ \\
\hline AW-Cyl-1-1 & {$[ \pm 45,0,90]_{\mathrm{s}}$} & 184.1 & 134.2 & 120 \\
AW-Cyl-2-1 & {$[ \pm 45, \mathrm{c} 45]_{2 \mathrm{~s}}$} & 436.3 & 329.2 & $\begin{array}{c}\text { el: } 350 \\
\text { el-pl: } 305\end{array}$ \\
AW-Cyl-3-1 & {$[ \pm 45,0,90]_{2 \mathrm{~s}}$} & 745.9 & 657.5 & 505 \\
AW-Cyl-4-1 & {$\left[ \pm 45,0_{4}, \mp 45\right]_{\mathrm{s}}$} & 621.4 & 558.6 & 465
\end{tabular}




$\begin{array}{llllc}\text { AW-Cyl-5-1* } & {\left[ \pm 45,90_{4}, \mp 45\right]_{\mathrm{s}}} & 672.7 & 407.9 & 420 \\ \text { AW-Cyl-11-1 } & {[ \pm 45,0,90]_{2 \mathrm{~s}}} & 745.9 & 676.6 & 510 \\ \text { AW-Cyl-92-01 } & {\left[ \pm 45,0_{2}\right]_{\mathrm{s}}} & 133.1 & 123.6 & 115 \\ \text { AW-Cyl-92-02 } & {\left[ \pm 45,90_{2}\right]_{\mathrm{s}}} & 170.1 & 142.0 & 95 \\ \text { AW-Cyl-92-03 } & {[ \pm 45,0,90]_{\mathrm{s}}} & 184.1 & 152.0 & 120 \\ \text { C1 } & {\left[\mp 45,0_{2}\right]_{\mathrm{s}}} & 133.9 & 123.5 & 115 \\ \text { C2 } & {\left[\mp 45,90_{2}\right]_{\mathrm{s}}} & 164.4 & 141.9 & 95 \\ \text { C3 } & {[\mp 45,0,90]_{\mathrm{s}}} & 185.5 & 152.1 & 120\end{array}$

*Damaged before testing

** strong material nonlinearity occurred, simulation has been rerun with elastic plastic material law

Table 5: Buckling loads and design loads for the composite shells tested at NASA LaRC (see [6] and [35])

The aluminum cylinders NTA tested at NASA LaRC, which have been considered for the studies shown in the previous sections, have been manufactured from a single sheet that is bended and fixed by a double-overlap splice joint [24]. Though these cylinders are not made of composite material, they are mentioned here, because they served as an isotropic reference case for the studies presented. Due to plastic deformations in the postbuckling regime, the cylinders could only be tested once up to collapse. All tests were carried out in presence of a perturbation load. The SPLA design load was found to be $N_{1}=187 \mathrm{kN}$ for a perturbation load of $P_{1}=70 \mathrm{~N}$. The minimum collapse load in test equals $162.8 \mathrm{kN}$, where the shell was tested in presence of a perturbation load of $65 \mathrm{~N}$.

For the shells of the German Aerospace Center (Deutsches Zentrum für Luft- und Raumfahrt, DLR) as well as for NTA, studies have been executed to determine the critical axial position of the perturbation load within the SPLA. For all shells the center turned out to yield the lowest design load.

Most composite shells considered have a symmetric layup and therefore, membrane forces and bending moments are decoupled. In order to depict the influence of the B-Matrix, the SPLA is applied to a set of shells tested by Tennyson and Muggeridge [36]. The dimensions of these cylinders are $L=315.5 \mathrm{~mm}$ and $R=159 \mathrm{~mm}$. For the thickness, only measured values are given in [36], which have an order of magnitude of $t \approx 0.7 \mathrm{~mm}$ for the whole laminate.

\begin{tabular}{clccc}
\hline \hline Name & Laminate & $\begin{array}{c}\text { Perf. BL in kN, } \\
\text { from [36] }\end{array}$ & $\begin{array}{c}\text { Experimental BL in kN, } \\
\text { from [36] }\end{array}$ & $\begin{array}{c}\text { SPLA } \\
N_{1} \text { in kN }\end{array}$ \\
\hline 1a & {$[-70,70,0]$} & 38.4 & 26.0 & 23.8 \\
2a & {$[-20,20,90]$} & 39.9 & $\mathbf{2 4 . 6}$ & $\mathbf{2 6 . 2}$ \\
3b & {$[90,90,90]$} & 33.9 & $\mathbf{2 2 . 6}$ & $\mathbf{2 6 . 7}$ \\
4a & {$[90,-45,45]$} & 36.8 & 24.8 & 22.9 \\
4b & {$[90,-45,45]$} & 36.1 & 22.6 & 22.4 \\
5b & {$[90,0,90]$} & 33.3 & $\mathbf{2 3 . 0}$ & $\mathbf{2 7 . 1}$ \\
\hline \hline
\end{tabular}




\begin{tabular}{clccc}
\hline $6 \mathrm{~b}$ & {$[45,0,-45]$} & 30.0 & 22.9 & 21.8 \\
$7 \mathrm{~b}$ & {$[-45,45,90]$} & 33.1 & 24.9 & 23.0 \\
$9 \mathrm{a}$ & {$[0,-45,45]$} & 32.4 & 25.9 & 20.5 \\
$9 \mathrm{~b}$ & {$[0,-45,45]$} & 33.7 & 24.7 & 21.3 \\
$11 \mathrm{a}$ & {$[30,90,30]$} & 36.2 & 26.1 & 20.5 \\
$11 \mathrm{~b}$ & {$[30,90,30]$} & 36.1 & $27.7^{*}$ & 20.4 \\
$12 \mathrm{a}$ & {$[30,90,-30]$} & 36.2 & 25.4 & 22.5 \\
$12 \mathrm{~b}$ & {$[30,90,-30]$} & 39.2 & 25.2 & 23.8 \\
\hline \hline
\end{tabular}

*Without thrust bearing experimental $\mathrm{BL}=22.0 \mathrm{kN}$

Table 6: Buckling loads and design loads for the composite shells tested by Tennyson and Muggeridge (see [36])

For the shells highlighted in bold letters in Table 6, the lower bound given by the SPLA exceeds the experimentally determined buckling load.

For most shells considered the SPLA provides a conservative lower bound. It is anticipated in the DESICOS project, and indicated by the study given in [19], that the SPLA only covers the effect of geometric imperfections. For some shells, this effect of geometric imperfections seems to be predominant, and the SPLA provides a reliable lower bound. However, it is not known in advance for which shells this applies.

\section{Influence of Coupling Terms on the SPLA}

As shown in section 2.2 and in [20], for some shells the SPLA yields only a small knockdown, which does not cover the test result. In some cases, the SPLA provides a reliable lower bound, but when reversing the stacking sequence, $N_{1}$ exceeds the test results, or is very close to it (e.g., Z32/Z33 and Z07/Z09). Obviously something changes in the coupling between axial load and bending, which causes the change in the behavior. Looking at the ABD matrix in

$$
\left(\begin{array}{l}
\mathbf{N} \\
\mathbf{M}
\end{array}\right)=\left(\begin{array}{ll}
\mathbf{A} & \mathbf{B} \\
\mathbf{B} & \mathbf{D}
\end{array}\right)\left(\begin{array}{l}
\boldsymbol{\varepsilon} \\
\mathbf{\kappa}
\end{array}\right)
$$

it is obvious that this coupling is expressed by the B matrix (for more explanation on laminate theory the reader is referred, e.g., to [37]).

Looking for instance at the ABD matrices of $\mathrm{Z} 07$ and $\mathrm{Z} 09$, the only difference are the signs of $B_{11}, B_{22}, B_{12}, B_{33}, D_{13}$ and $D_{23}$.

In order to get some insight on how these coupling terms influence the SPLA, a study is carried out in which the ABD matrix of a cylinder is varied continuously between the ones of Z07 and Z09, according to

$$
\mathbf{A B D}=\delta_{B} \mathbf{A B D}_{\text {Z07 }}+\left(1-\delta_{B}\right) \mathbf{A} \mathbf{B D}_{\text {Z09 }}
$$

with $\delta_{B}=0, \ldots, 1$. For $\delta_{B}=0.5, B_{11}=B_{22}=B_{12}=B_{33}=D_{13}=D_{23}=0$ applies.

The geometric dimensions used for the study are the ones of Z07 and Z09: $\mathrm{L}=500, \mathrm{R}=250$. 
For the following studies, the material properties given by Hühne et al. [7] have been used, which slightly differ from the ones given in [32].

\subsection{Finite Element Study}

Using the finite element model described in Appendix B, the eigenvalues given in Table 7 are determined considering the perfect cylinder. The results show that the buckling load increases from $\delta_{B}=0$ to $\delta_{B}=1$ with a maximum at $\delta_{B}=0.9$. For the Z09 and Z07 configurations, the results in Table 7 slightly differ from the ones given in Table 3, because Hühne used an accompanying eigenvalue analysis in a nonlinear simulation to determine the buckling load of the perfect shell (for details see [33]).

\begin{tabular}{cccccccc}
\hline \hline$\delta_{B}$ & $0(\mathrm{Z} 09)$ & 0.2 & 0.4 & 0.6 & 0.8 & 0.9 & $1(\mathrm{Z} 07)$ \\
\hline $\mathrm{BL}$ & 17.8 & 22.5 & 26.9 & 30.9 & 34.6 & 36.3 & 34.7 \\
\hline
\end{tabular}

Table 7: Buckling loads (eigenvalues) obtained by linear eigenvalue analysis for cylinders with B matrix interpolated between cylinders $\mathrm{Z07}$ and $\mathrm{Z09}$

The same model is then used to determine the lower bound $N_{1}$ given by the SPLA, where now a nonlinear simulation is performed. In Figure 13 the buckling load is plotted over the perturbation load for $\delta_{B}=0$ (Z09), $\delta_{B}=0.5$ and $\delta_{B}=1$ (Z07). For the case of $\delta_{B}=0.5, N_{1}$ is higher than for Z07, though the buckling load of the perfect shell is higher for Z07. Compared to these two configurations, the buckling loads of Z09 are on a significantly lower level and also the change of buckling load is significantly smaller.

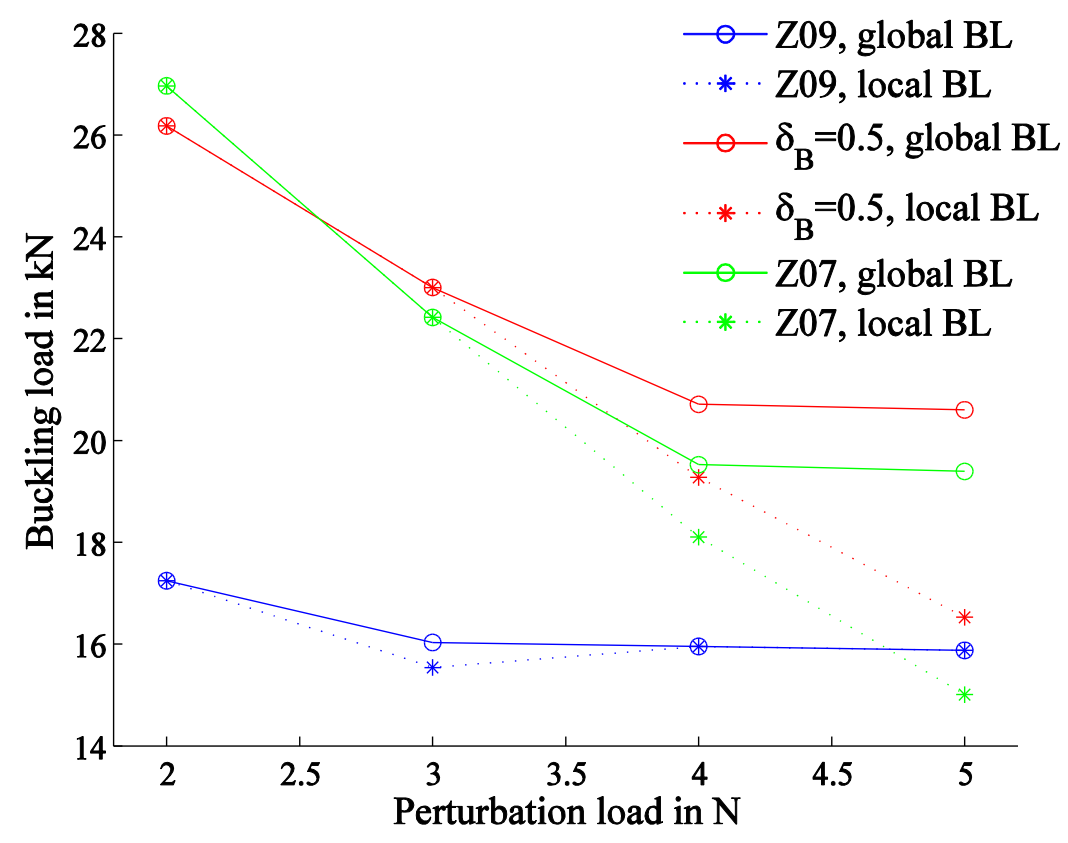

Figure 13: Buckling load over perturbation load for different values of $\delta_{B}$ 
In order to get a better overview how the buckling load and the knockdown changes with varying ABD matrix, the buckling loads are plotted over $\delta_{B}$ in Figure 14. For low values of $\delta_{B}$ the buckling load is hardly affected by the perturbation load and consequently, the load bound $\mathrm{N}_{1}$ is close to the buckling load of the undisturbed cylinder. For higher values of $\delta_{B}$ the sensitivity increases significantly such that the maximum for the design load $N_{1}$ (for $\mathrm{P}=4 \mathrm{~N}$ ) is found for $\delta_{B}=0.6$, while the maximum buckling load of the undisturbed cylinder was found for $\delta_{B}=0.9$. (For $\delta_{B}=0.4$ and $\mathrm{P}=5$ no local buckling load occurs, which is the reason for the kink in the dashed pink curve.)

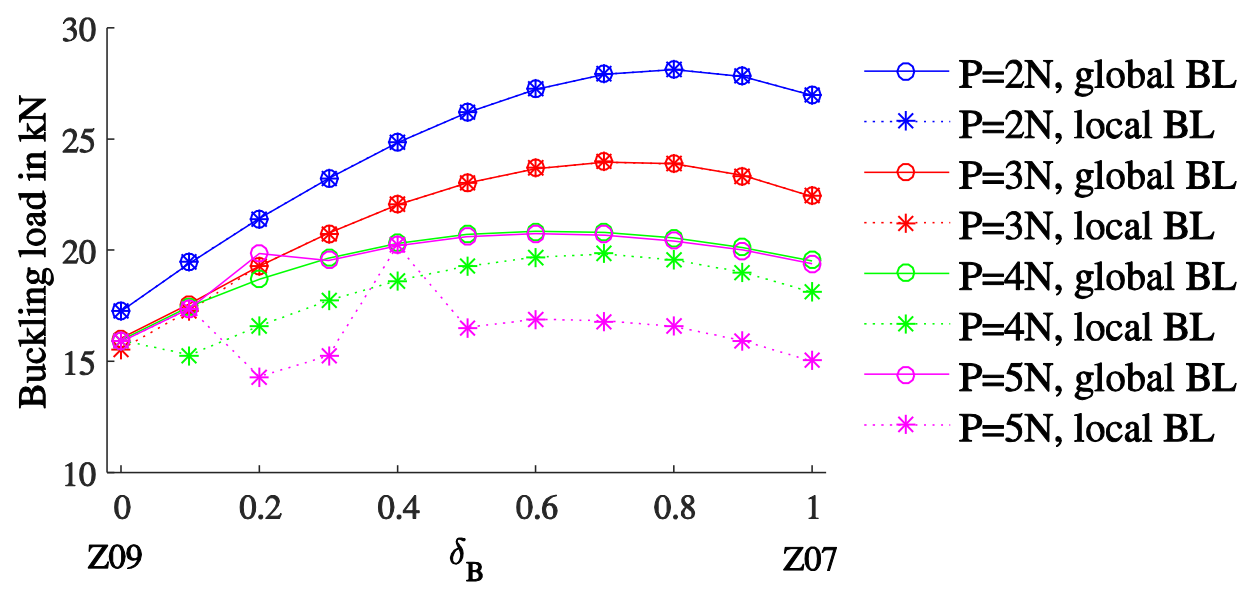

Figure 14: Global and local buckling load over $\delta_{B}$ given by FEM

\subsection{Semi-Analytical Study}

An indication of the imperfection sensitivity of a shell can be obtained with the help of Koiter's initial post-buckling theory. In particular, in this section the influence of the ABD matrix on the imperfection sensitivity of the shell is investigated. In the single mode formulation of Koiter's initial post-buckling theory, denoted as "b-factor method" in the following, the imperfection sensitivity of one specific buckling mode, usually the first buckling mode (i.e., the one corresponding to the lowest buckling load) or one of the first few buckling modes is analyzed. A high degree of imperfection sensitivity corresponds to those cases in which a large decrease in buckling load occurs for the imperfect structure, when an imperfection in the shape of the buckling mode is present. For details on the semi-analytical implementation of the b-factor method one is referred to Appendix A. Similar to the Finite Element studies in the previous section, in this section the b-factor method is applied for studying the effect of varying the ABD matrix between the Z07 and Z09 properties on the imperfection sensitivity of the shell. In this way, one can use Koiter's b-factor method to identify in which cases the SPLA should be used with caution. 


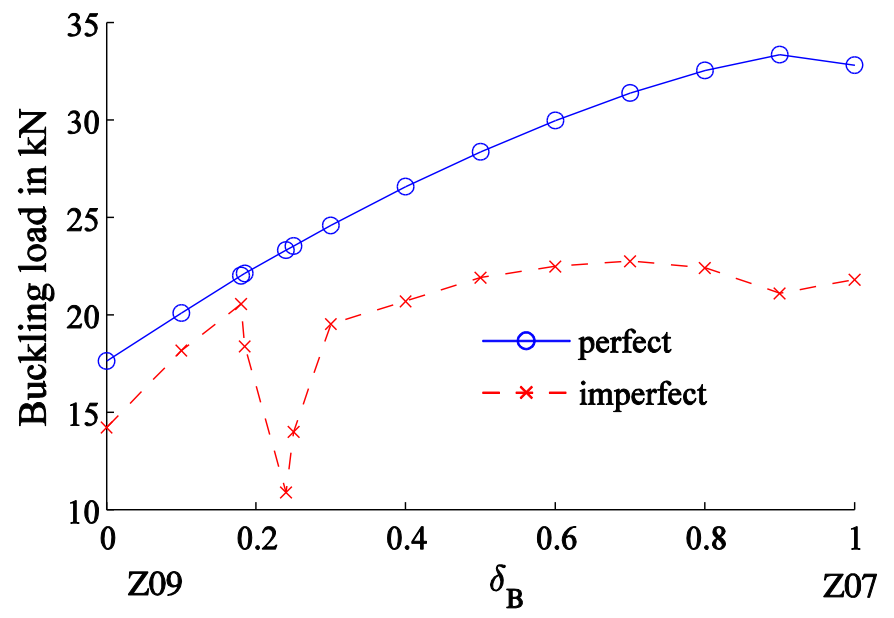

Figure 15: Buckling loads for varying ABD Matrix given by semi-analytical approach

In Figure 15 the bifurcation buckling loads are shown, obtained in an eigenvalues analysis of the perfect shell during loading. The bifurcation buckling loads of the Z09 shell and the Z07 shell significantly differ.

The semi-analytical bifurcation buckling analysis corresponds to the bifurcation points on the axisymmetric nonlinear fundamental state. The buckling load levels show a trend similar to the results of the finite element linear buckling eigenvalue analyses.

The nonlinear buckling loads are calculated using Koiter's theory. The approach gives an estimate of the reduction in the load carrying capability of the shell. In order to determine the limit-point buckling load of the imperfect cylinder, an affine imperfection with an amplitude of $\bar{\xi}=0.25$ (i.e. $25 \%$ of the wall thickness) is used. In Figure 16 the knock-down factors corresponding to this imperfection form and amplitude are shown. In Figure 15 the corresponding limit-point buckling loads have been plotted.

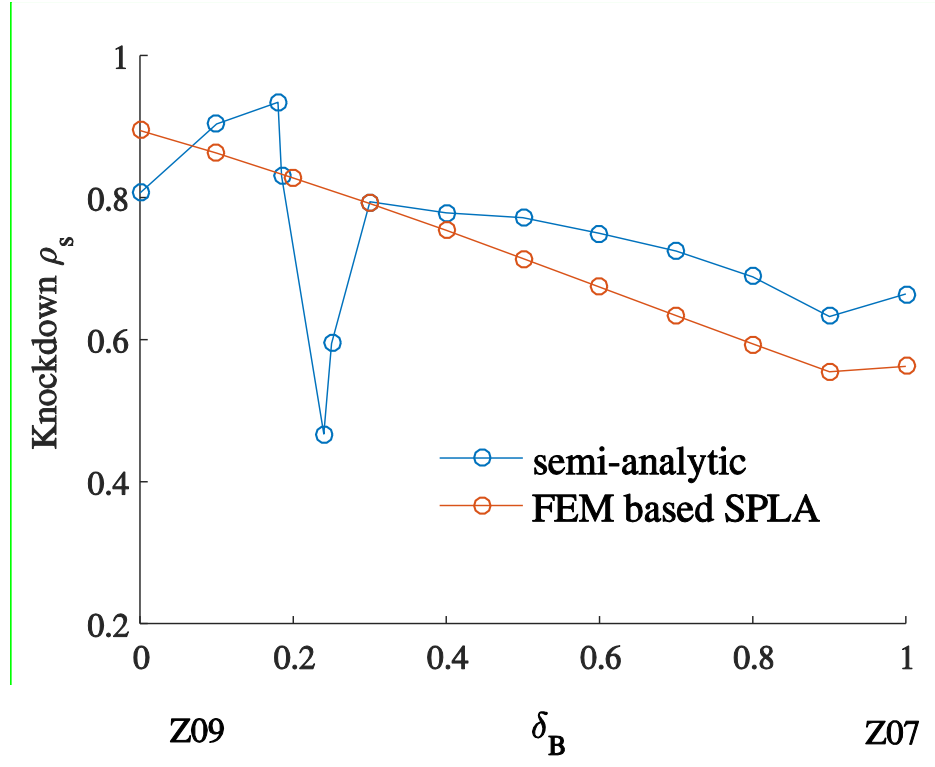


The results of the semi-analytical analysis for larger values of $\delta_{B}$ show the same tendency as the results of the SPLA analyses with large perturbation loads (compare Figure 14, P $=4 \mathrm{~N}$ ). It is seen that for values of $\delta_{B}$ between 0.3 and 0.9 , a considerable imperfection sensitivity occurs, which increases gradually for increasing values of $\delta_{B}$ (the knockdown $\rho_{s}$ decreases from 0.79 for $\delta_{B}=$ 0.3 to 0.63 for $\left.\delta_{B}=0.9\right)$. For the Z07 geometry, $\rho_{s}=0.66$. Also the Z09 geometry $\left(\delta_{B}=0.0\right)$ corresponds to a considerable imperfection sensitivity $\left(\rho_{s}=0.81\right)$. It is further observed, that for the buckling modes occurring in a specific small range of shell configurations close to $\delta_{B}=0.2$, the semi-analytical b-factor approach predicts a very high imperfection sensitivity, resulting in a large drop of the knock-down factor. It is interesting to note, that for this range of configurations also the SPLA predicts a large reduction in buckling load as compared to the linear buckling load for high perturbation loads $(\mathrm{P}=5 \mathrm{~N})$, when considering the local buckling case (Figure 14).

In the case of shell Z07, the degree of imperfection sensitivity predicted by the SPLA and by Koiter's b-factor method are in agreement. The strong imperfection sensitivity for Z07, predicted by SPLA (the SPLA design load corresponding to a reduction of $36 \%$ as compared with the linear buckling load), and also observed in experiments, is also predicted by Koiter's b-factor method (a reduction of 34\% as compared with the bifurcation buckling load). For the case of shell Z09 however, the b-factor method predicts a considerable imperfection sensitivity (a reduction of $19 \%$ as compared with the bifurcation buckling load), whereas the SPLA results in a design load that is only moderately decreased (corresponding to a reduction of $11 \%$ as compared with the linear buckling load) and does not predict the degree of imperfection sensitivity observed in the experiment. A comparison of the results of the two approaches for the Z09 shell therefore confirms that in this case the result of the SPLA should be considered with caution.

In Figure 17 and Figure 18, the axisymmetric nonlinear prebuckling deformation modes and buckling modes are shown for both the Z09 and for the Z07 shell. The Z09 shell shows a pronounced short wave prebuckling and buckling deformation over the full shell length. In contrast with the behavior of the Z09 shell, the prebuckling deformation of the Z07 shell $\left(\delta_{B}=1\right)$ shows a region with bending at the boundaries, which decays towards the center of the shell.

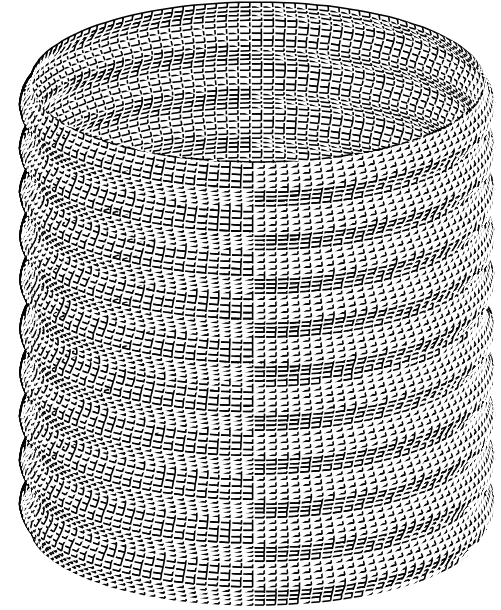

(a) prebuckling deformation

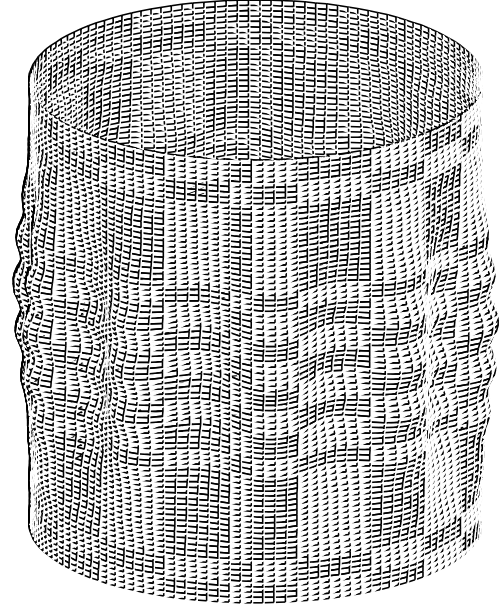

(b) buckling mode

Figure 17: (a) Nonlinear prebuckling deformation (axisymmetric) and (b) buckling mode of shell Z09 for buckling load corresponding to $n=10$ circumferential waves (lowest buckling load) 
Observation of the characteristics of the prebuckling and buckling deformation of the Z09 shell reveals that the shell in this case tends to buckle in a short wave axi-symmetric pattern. In the semi-analytical b-factor approach, which includes a nonlinear pre-buckling state, this is reflected in the significant growth of a short-wave axi-symmetric prebuckling deformation pattern at load levels near the bifurcation buckling load.

For this specific case - a configuration for which the shell tends to buckle with an axisymmetric pattern - a considerable imperfection sensitivity exists, which is not predicted by the SPLA. In general, by comparing the degree of imperfection sensitivity predicted by the two approaches, Koiter's b-factor method can be used to identify parameter ranges, in which the SPLA should be used with caution.

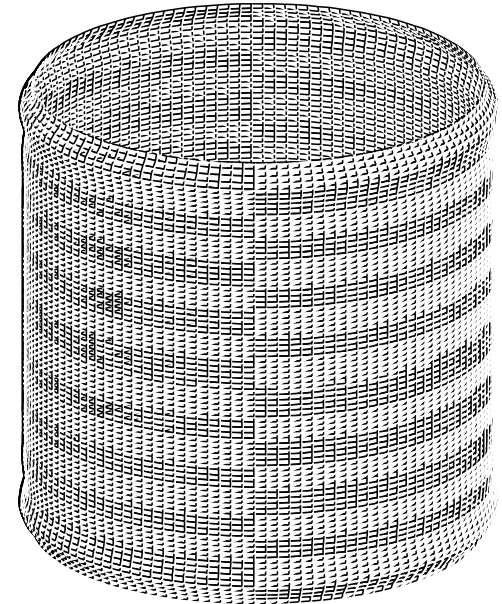

(a) prebuckling deformation

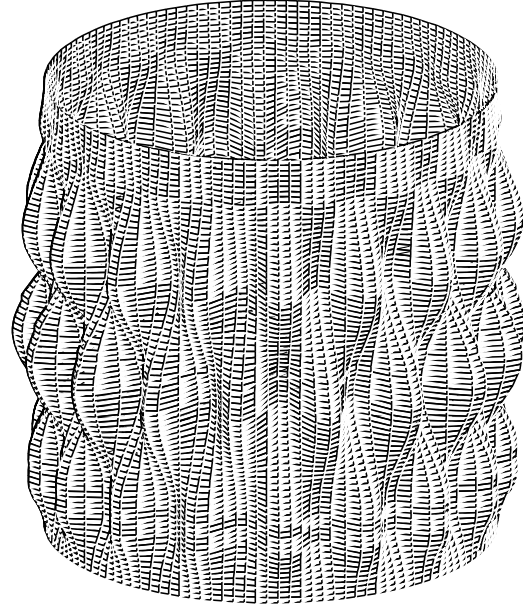

(b) buckling mode, $n=17$

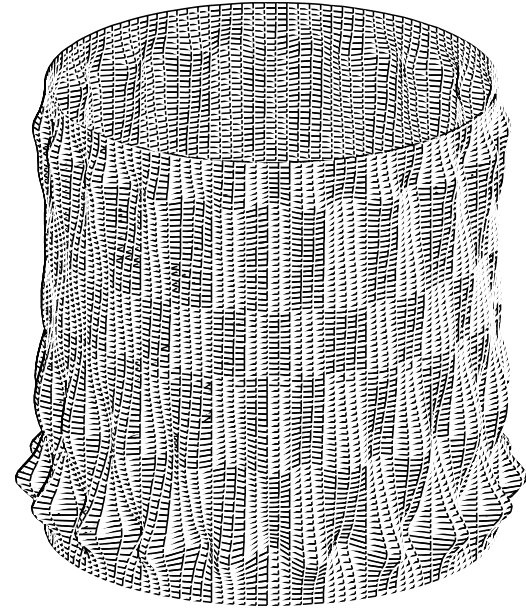

(c) buckling mode, $n=18$

Figure 18: (a) Nonlinear prebuckling deformation (axisymmetric) of shell Z07, (b) buckling mode for first buckling load corresponding to $n=17$ circumferential waves and (c) for second buckling load corresponding to $n=18$ circumferential waves

\subsection{Analytical Considerations}

Using a purely analytical approach Geier et al. [21] found a similar result for the shells Z32 and Z33, as given in the previous chapter for Z07 and Z09 (in terms of buckling load and mode). For deriving a closed form solution of the buckling load of a perfect shell, Geier et al. assumed a linear, homogenous prebuckling state, classical "S3" boundary conditions and orthotropy $\left(B_{13}=B_{23}=D_{13}=D_{23}=0\right)$. The approach provides different expression for the buckling load when considering only axisymmetric buckling modes (hence without waves in circumferential direction) or asymmetric buckling modes (number of circumferential waves $>0$ ). The solution for the axisymmetric buckling load (3) given in [21] gives an idea about which types of shells tend to buckle in an axisymmetric mode and not in an asymmetric mode.

$$
F_{b}=\frac{-4 \pi}{a_{22}}\left(\sqrt{\tilde{D}_{11} a_{22}+e_{21}^{2}}+e_{21}\right)
$$

Here, $a_{22}$ is an entry of the inverse plain stiffness matrix $\mathbf{a}=\mathbf{A}^{-1}, e_{21}$ is an entry of the eccentricity matrix $\mathbf{e}=\mathbf{a} \mathbf{B}$, and $\tilde{D}_{11}$ is an entry of the modified bending stiffness matrix 
$\tilde{\mathbf{D}}=\mathbf{D}-\mathbf{B}^{T} \mathbf{A}^{-1} \mathbf{B}$. (Geier et al. assumed the stacking direction from outside to inside, which differs from the convention used in section 2.2. This has an impact on the sign of $e_{21}$ !) Geier et al. concluded that the sign and magnitude of the term $e_{21}$ determines whether a cylinder tends buckle in an axisymmetric or an asymmetric mode.

Determining the lowest buckling load $F_{b}$ with axisymmetric buckling mode and the eccentricity term $e_{21}$ for varying $\mathrm{ABD}$ matrix according to equation (3) yields the functions shown in Figure 19 and Figure 20.

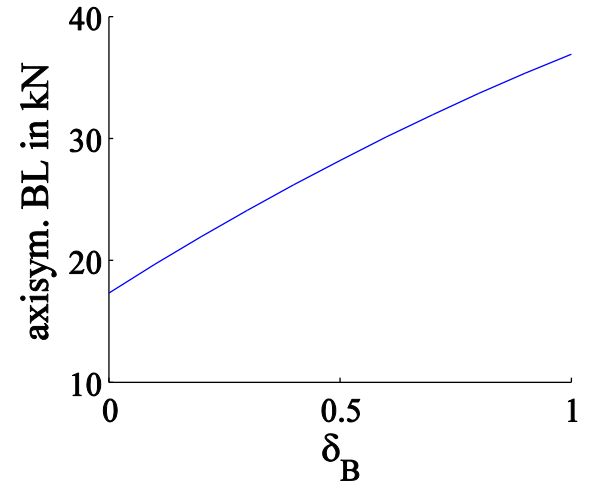

Figure 19: Analytically determined buckling load for axisymmetric modes over $\delta_{\mathrm{B}}$

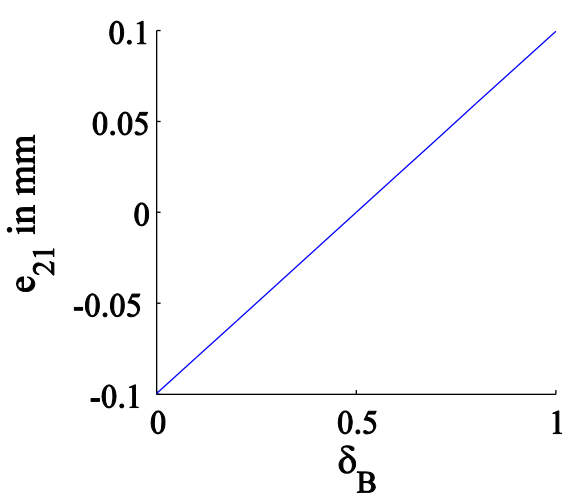

Figure 20: Eccentricity $e_{21}$ over $\delta_{B}$

Since this analytical approach considers the perfect cylinder it does not provide information on the imperfection sensitivity. Also, the boundary conditions ("S3") differ from the ones usually applied in test (clamped). Still, the analytical approach indicates whether a shell tends to buckle axisymmetric or asymmetric, while being very easy to apply.

For Z07 and Z09 as well as for Z32 and Z33 it has been observed that the shells that tend to buckle axisymmetric are the ones where the SPLA was not conservative. Therefore, it is proposed to use the term $e_{21}$ as an indicator, if the SPLA works for a shell considered. In Figure 21 , the ratio of lower bound given by SPLA and experimental result is plotted over the eccentricity $e_{21}$, normalized with the wall thickness $t$. The test results above the red line are the cases in which the SPLA is not conservative.

Figure 21 shows the tendency that for increasing $e_{21} / t$, the ratio of SPLA and experiment decreases. In other words, the likelihood that an experimental test result is lower than the $N_{1}$ increases as $e_{21} / t$ (and thereby the tendency to buckle axisymmetrically) decreases. Still, it cannot be concluded in general that the SPLA is always conservative for a certain value of $e_{21} / t$. Especially for $e_{21} / t=0$ a lot of test results are found for which the ratio of SPLA lower bound $\left(N_{1}\right)$ and experiment is above 1 . This is due to the fact the buckling load is subject to large scatter caused by various effects. Still, the ratio $e_{21} / t$ indicates if the SPLA is likely to giving a conservative lower bound or not. 


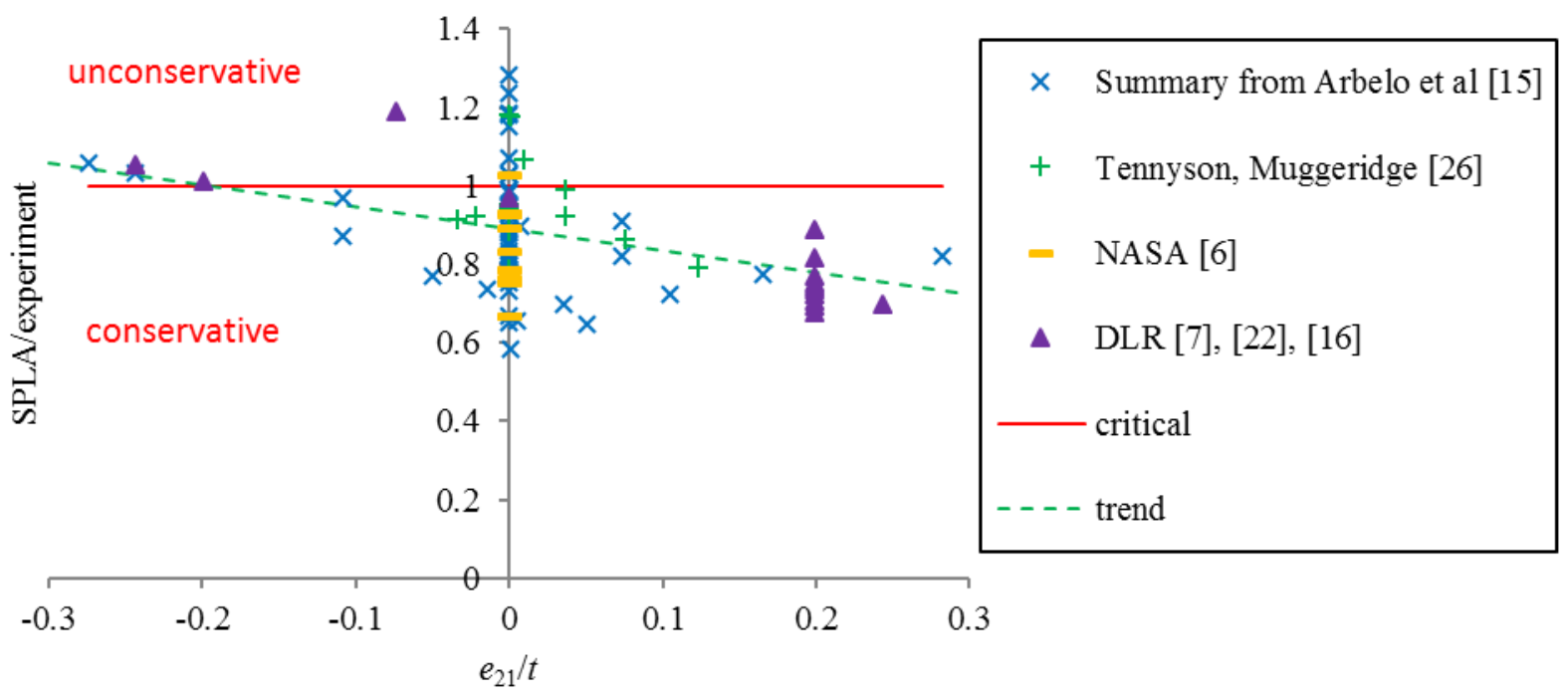

Figure 21: Ratio of SPLA lower bound and test result of normalized eccentricity

\section{Conclusions}

In the present paper, two main issues have been addressed, that make it possible to better appreciate the potential and the limits when applying the SPLA. It is noted that the findings of this paper apply to axially compressed cylinders, for which the loading is displacement driven. Based on the observations by Friedrich and Schröder [18], the applicability of the SPLA to load driven scenarios needs to be further analyzed and discussed.

Firstly, the question whether a single perturbation load in general represents a "worst case" imperfection has been discussed. The second important issue, the influence of the stiffness properties of the shell on the SPLA predictions, has been thoroughly investigated.

With regard to the first issue, results of applying the single perturbation load approach (SPLA) to various shells have been summarized. For selected shells, the SPLA is compared to the effect of other locally concentrated imperfections, showing that the SPLA covers the effect of local boundary imperfections, and single buckles. By increasing the number of perturbation loads applied at the same time, it was shown that the lower bound obtained by this multiple perturbation load approach decreases with the number of perturbation loads. This should be kept in mind when applying the SPLA, since it shows that the single perturbation load is not the worst case imperfection. However, with increasing number of perturbation loads the induced imperfection becomes less realistic.

Concerning the second issue, a summary of results of the SPLA applying to numerous cylinders show that some cylinders seem to be less sensitive to local imperfections than others. In tests, the buckling load of these cylinders is smaller than the design load $N_{1}$, because other effects (e.g., load eccentricity, thickness variations, ...) become predominant. The applicability of the SPLA seems to depend significantly on the coupling terms in the ABD matrix. In order to analyze this observation, the buckling behavior is investigated for varying ABD matrix. 
A semi-analytical formulation of Koiter's b-factor method has been used to assess the applicability of the SPLA by a comparison of the imperfection sensitivity predictions of the two approaches. The b-factor method gives an estimate of the imperfection sensitivity and can also provide an estimate of the decrease of the load carrying capability of the shell for a given imperfection.

The investigations in this paper have shown that in certain cases in which the SPLA does not give appropriate results, the "perfect" shell shows a pronounced short wave prebuckling and buckling deformation. In these cases the single buckle resulting from the SPLA approach may not be a representative worst-case imperfection for the actual shell buckling behavior and might therefore not be able to predict the imperfection sensitivity appropriately. For these specific cases configurations for which the shell tends to buckle with an axisymmetric pattern - a considerable imperfection sensitivity exists, which is not predicted by the SPLA.

The semi-analytical Koiter-type approach also gave a clue for the definition of a useful practical indicator, the value of the stiffness parameter $e_{21}$, for identification of cases in which the SPLA should be used with caution. In [21] it was shown, that axisymmetric buckling occurs for shells with a stiffness parameter $e_{21}$ which is negative. Since the tendency to buckle in an axisymmetric pattern is reflected by the stiffness parameter $e_{21}$, in the present work it is proposed to use this stiffness parameter $e_{21}$ as a simple, practical indicator to identify cases in which the SPLA should be applied with caution. This indicator can be directly computed from the ABD matrix. This coefficient shows whether a cylinder tends to buckle axisymmetrically and therefore, whether applying the SPLA requires a "closer look". Looking at the SPLA versus test results with respect to $e_{21} / t$ indeed shows the tendency that with decreasing $e_{21} / t$, the SPLA fails more often. It is however not believed that a correction to the knockdown factor obtained from the SPLA can be directly defined as function of $e_{21}$, as there is still too much scatter in the buckling load. The bfactor method can be helpful in establishing such a correction factor to the knockdown factor obtained from the SPLA.

\section{Appendix A - Semi-Analytic imperfection sensitivity analysis}

The semi-analytical method that is used in this study is capable of accurately taking into account the effect of boundary conditions at the shell edges [38]. Koiter's initial postbuckling theory, including the effect of a nonlinear prebuckling state, is applied to analyse the imperfection sensitivity. The analysis is based on Donnell-type governing equations. A Fourier decomposition of the solution is used in the circumferential direction of the shell in order to obtain a set of ordinary differential equations for the length direction. Subsequently, the resulting two-point boundary value problem is solved numerically by means of the parallel shooting method.

Bifurcation buckling calculations have been performed for a nonlinear prebuckling state. The reduction of the load carrying capability of the shell in the case of asymmetric imperfections is evaluated via initial-postbuckling and imperfection sensitivity analysis, in the following denoted as the b-factor method.

The b-factor method gives information about the initial curvature of the load versus deflection curve of the structure without 'asymmetric' imperfections. In the presence of asymmetric initial imperfections, buckling occurs at a limit-point in the load versus deflection curve. In a single mode analysis, for imperfect shells the following expansion is used for the load parameter $\Lambda$,

$$
\left(\Lambda-\Lambda_{c}\right) \xi=a \Lambda_{c} \xi^{2}+b \Lambda_{c} \xi^{3}+\ldots-\alpha \Lambda \bar{\xi}-\beta\left(\Lambda-\Lambda_{c}\right) \bar{\xi}+O(\xi \bar{\xi})
$$


where $\bar{\xi}$ corresponds to the normalized amplitude of an asymmetric initial imperfection, and the imperfection form factors $\alpha$ and $\beta$ depend on the assumed imperfection pattern [38]. Further, $\bar{\xi}$ has been normalized with respect to the shell thickness $h$. For "symmetric" structures (the first post-buckling coefficient $a=0$ ), the reduction of the load carrying capacity with respect to the bifurcation buckling load can be estimated using the modified Koiter formula [39],

$$
\left(1-\rho_{s}\right)^{\frac{3}{2}}=\frac{3}{2} \sqrt{-3 \alpha^{2} b}\left[1-\frac{\beta}{\alpha}\left(1-\rho_{s}\right)\right]|\bar{\xi}|
$$

where

$$
\rho_{s}=\frac{\Lambda_{s}}{\Lambda_{c}}
$$

is the ratio between the limit-point buckling load $\Lambda_{s}$ and the bifurcation buckling load $\Lambda_{c}$. For the case of axial compression $\Lambda=P, \Lambda_{c}=P_{c}$, and $\Lambda_{s}=P_{s}$.

The limit-point buckling load in the case of axial compression is obtained from

$$
P_{s}=\rho_{s} P_{c}=\rho_{s} \lambda_{c} P_{c l}
$$

In this study, an imperfection with the same shape as the buckling mode, referred to as affine imperfection,

$$
\frac{\bar{W}}{h}=\frac{W^{(1)}}{h}=\bar{\xi}\left[w_{1}(x) \cos \left(\frac{n y}{R}\right)+w_{2}(x) \cos \left(\frac{n y}{R}\right)\right]
$$

is used in the calculations, where $W^{(1)}(x, y)$ is the buckling mode, $\bar{W}(x, y)$ denotes the initial imperfection, $\bar{\xi}$ is the imperfection amplitude, $h$ denotes the wall thickness, and $x$ and $y$ correspond to the axial and the circumferential coordinate, respectively Figure 22.

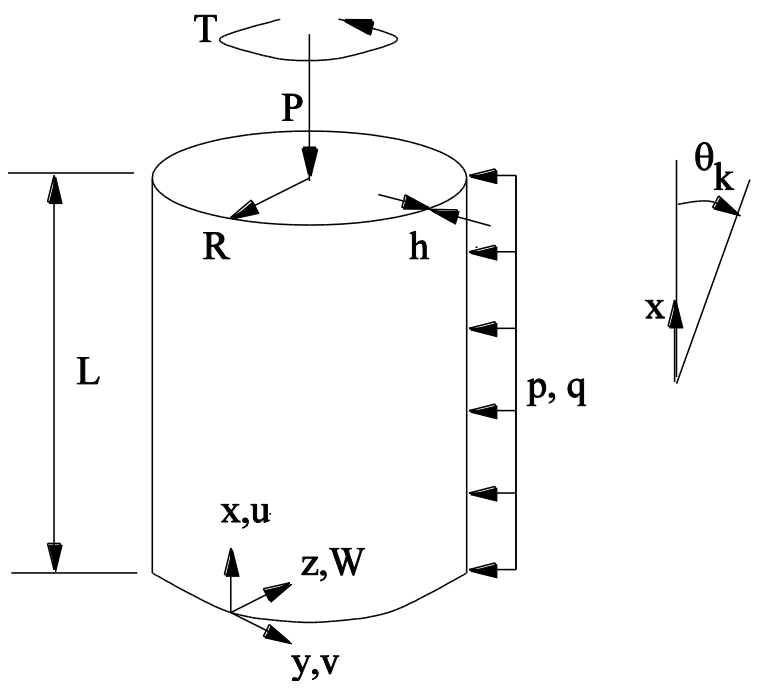

Figure 22: Shell geometry, coordinate system and applied loading 


\section{Appendix B - Buckling Analyses with ABAQUS}

The finite element buckling analyses have been performed with the commercial code ABAQUS. The models used linear shells elements with reduced integration (S4R). In most cases, the mesh consists of 61 nodes in axial direction and the number of nodes in circumferential direction was chosen to obtain square shaped elements.

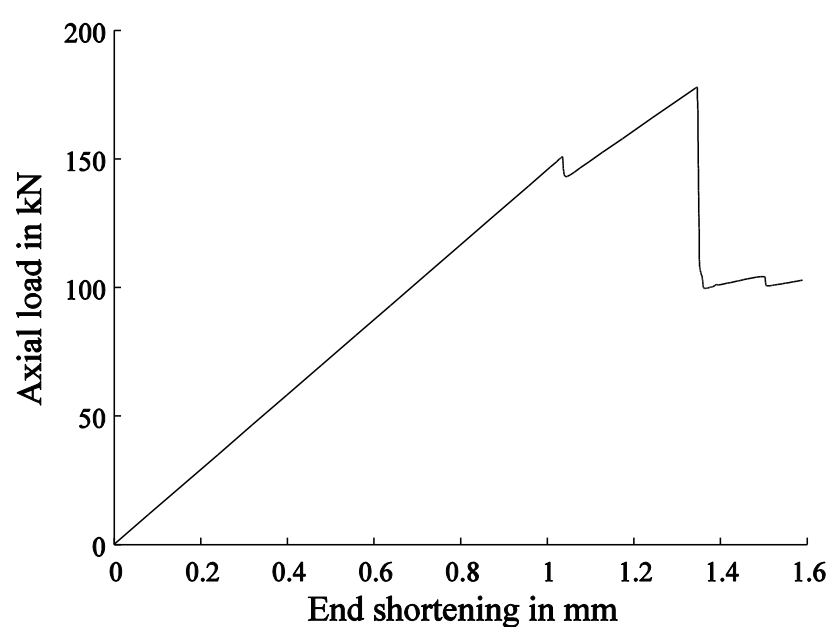

Figure 23: Load displacement curve obtained from finite element simulation of a cylinder with perturbation load

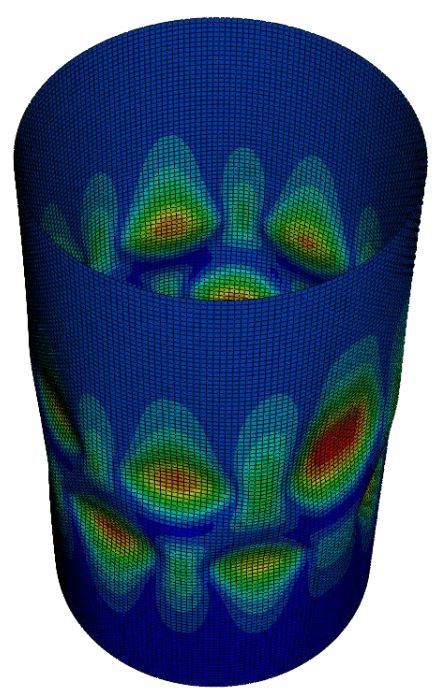

Figure 24: Finite element model of cylinder NTA with postbuckling deformations

In order to capture drops in the load displacement curve as shown in Figure 23, the non-linear simulations have been run displacement driven with artificial damping (*STATIC, STABILIZE). As all cylinders considered had clamped edged in tests, also clamped ends were used as boundary conditions in the model.

\section{Appendix C - Overview of shells considered}

In Table 8 the eccentricity term (see section 3.3) is given for the shells mentioned in section 2.2. The cylinders tested at NASA LaRC (AW-Cyl -...) all have a symmetric lay-up and therefore $e_{21}=0$ for these shells.

\begin{tabular}{cccccc}
\hline Shell ID & Lay-up & $e_{21}$ & $\begin{array}{c}\text { Experimental } \\
\text { buckling load in } \\
\mathrm{kN}\end{array}$ & $N_{1}$ in $\mathrm{kN}$ & Ref. \\
\hline Z07 & {$[ \pm 24, \pm 41]$} & $9.96 \mathrm{E}-05$ & 21.3 & 17.4 & {$[7]$} \\
Z09 & {$[ \pm 41, \pm 24]$} & $-9.96 \mathrm{E}-05$ & 15.7 & 14.7 & \\
Z10 & {$[24, \pm 41,-24]$} & $-1.22 \mathrm{E}-20$ & 15.7 & 13.8 \\
$\mathrm{Z} 12$ & {$[ \pm 45,0,-79]$} & $-3.69 \mathrm{E}-05$ & 18.6 & 20.2 & \\
\hline $\mathrm{Z} 32 *$ & {$\left[\mp 51, \mp 45, \mp 37, \mp 19,0_{2}\right]$} & -0.3046 & 89 & $\sim 94$ & {$[22]$}
\end{tabular}




\begin{tabular}{cccccc}
$\mathrm{Z33} *$ & {$\left[0_{2}, \pm 19, \pm 37, \pm 45, \pm 51\right]$} & 0.3046 & 173 & $\sim 121$ & \\
\hline $1 \mathrm{a}$ & {$[-70,70,0]$} & -0.022846 & 26 & 23.8 & {$[36]$} \\
$2 \mathrm{a}$ & {$[-20,20,90]$} & 0.0065166 & 24.6 & 26.2 & \\
$3 \mathrm{~b}$ & {$[90,90,90]$} & $4.7709 \mathrm{E}-18$ & 22.6 & 26.7 & \\
$4 \mathrm{a}$ & {$[90,-45,45]$} & 0.0257 & 24.8 & 22.9 & \\
$4 \mathrm{~b}$ & {$[90,-45,45]$} & 0.025421 & 22.6 & 22.4 & \\
$5 \mathrm{~b}$ & {$[90,0,90]$} & 0.0008189 & 23 & 27.1 & \\
$6 \mathrm{~b}$ & {$[45,0,-45]$} & -0.00023423 & 22.9 & 21.8 & \\
$7 \mathrm{~b}$ & {$[-45,45,90]$} & -0.015042 & 24.9 & 23 & \\
$9 \mathrm{a}$ & {$[0,-45,45]$} & 0.084503 & 25.9 & 20.5 & \\
$9 \mathrm{~b}$ & {$[0,-45,45]$} & 0.052073 & 24.7 & 21.3 & \\
$11 \mathrm{a}$ & {$[30,90,30]$} & $-6.0298 \mathrm{E}-18$ & 26.1 & 20.5 & \\
$11 \mathrm{~b}$ & {$[30,90,30]$} & $-1.3143 \mathrm{E}-18$ & $27.7 / 22.0^{*}$ & 20.4 & \\
$12 \mathrm{a}$ & {$[30,90,-30]$} & 0.000041883 & 25.4 & 22.5 & \\
$12 \mathrm{~b}$ & {$[30,90,-30]$} & $-9.7812 \mathrm{E}-19$ & 25.2 & 23.8 & \\
\hline \hline
\end{tabular}

Table 8: Buckling loads and eccentricity term for various shells

\section{Acknowledgement}

Part of the work was carried out during the first author's stay at NASA Langley Research Center. The support during that time, especially by Mark Hilburger, is gratefully acknowledged. The second and third author acknowledge the financial support received from the European Community's Seventh Framework Programme (FP7/2007-2013) under Priority Space, "DESICOS - New Robust DESIgn Guideline for Imperfection Sensitive COmposite Launcher Structures", Grant Agreement Number 282522.

\section{References}

[1] W. T. Koiter, "On the Stability of Elastic Equilibrium," NASA, NASA-TT-F-10833, 1967.

[2] H. Ohira, "Local Buckling Theory of Axially Compressed Cylinders," in Proceedings of the Eleventh Japan National Congress for Applied Mechanics, 1961, pp. 37-41.

[3] V. I. Weingarten, E. J. Morgan, and P. Seide, "Elastic Stability of Thin-Walled Cylindrical and Conical Shells Under Axial Compression," AIAA Journal, vol. 3, no. 3, pp. 500505, 1965.

[4] "Buckling of Thin-Walled Circular Cylinders," NASA, NASA SP-8007, 1968. 
[5] J. Arbocz and J. H. Starnes Jr, "Future Directions and Challenges in Shell Stability Analysis," Thin-Walled Structures, vol. 40, no. 9, pp. 729-754, 2002.

[6] J. Arbocz and M. W. Hilburger, "Toward a Probabilistic Preliminary Design Criterion for Buckling Critical Composite Shells," AIAA Journal, vol. 43, no. 8, pp. 1823-1827, 2005.

[7] C. Hühne, R. Rolfes, E. Breitbach, and J. Teßmer, "Robust Design of Composite Cylindrical Shells Under Axial Compression - Simulation and Validation," Thin-Walled Structures, vol. 46, no. 7-9, pp. 947-962, 2008.

[8] B. Kriegesmann, R. Rolfes, C. Hühne, and A. Kling, "Fast Probabilistic Design Procedure for Axially Compressed Composite Cylinders," Composites Structures, vol. 93, pp. 3140-3149, 2011.

[9] M. W. Hilburger, "Developing the Next Generation Shell Buckling Design Factors and Technologies," presented at the 53rd AIAA/ASME/ASCE/AHS/ASC Structures, Structural Dynamics and Materials Conference, Honolulu, USA, 2012, p. AIAA Paper 2012-1686.

[10] I. Elishakoff, S. van Manen, P. G. Vermeulen, and J. Arbocz, "First-Order SecondMoment Analysis of the Buckling of Shells with Random Imperfections," AIAA journal, vol. 25, no. 8, pp. 1113-1117, 1987.

[11] I. Elishakoff and J. Arbocz, "Reliability of Axially Compressed Cylindrical Shells With General Nonsymmetric Imperfections," Transactions of the ASME, vol. 52, pp. 122-128, 1985.

[12] J. Arbocz and J. M. A. M. Hol, "Collapse of Axially Compressed Cylindrical Shells with Random Imperfections," AIAA Journal, vol. 29, no. 12, pp. 2247-2256, 1991.

[13] E. L. Jansen, H. Abramovich, and R. Rolfes, "The direct prediction of buckling loads of shells under axial compression using VCT - towards an upgraded approach," in Proceedings of the 29th Congress of the International Council of the Aeronautical Sciences, St. Petersburg, Russia, 2014.

[14] M. A. Arbelo, K. Kalnins, O. Ozolins, E. Skukis, S. G. P. Castro, and R. Degenhardt, "Experimental and numerical estimation of buckling load on unstiffened cylindrical shells using a vibration correlation technique," Thin-Walled Structures, vol. 94, pp. 273-279, Sep. 2015.

[15] J. Singer, "Vibration correlation techniques for improved buckling predictions of imperfect stiffened shells," in Buckling of shells in Offshore Structures, J. Harding, P. Dowling, and N. Agelidis, Eds. London: Granada, 1982, pp. 285-329.

[16] J. Singer, "Vibrations and buckling of imperfect stiffened shells- Recent developments," in Collapse: The buckling of structures in theory and practice, J. Thompson and G. Hunt, Eds. Cambridge: Cambridge University Press, 1983, pp. 443-479.

[17] R. Degenhardt, S. G. P. Castro, M. A. Arbelo, R. Zimmerman, R. Khakimova, and A. Kling, "Future structural stability design for composite space and airframe structures," ThinWalled Structures, vol. 81, pp. 29-38, Aug. 2014.

[18] L. Friedrich and K.-U. Schröder, "Discrepancy between boundary conditions and load introduction of full-scale built-in and sub-scale experimental shell structures of space launcher vehicles," Thin-Walled Structures, vol. 98, Part B, pp. 403-415, Jan. 2016. 
[19] B. Kriegesmann, R. Rolfes, C. Hühne, J. Teßmer, and J. Arbocz, "Probabilistic Design of Axially Compressed Composite Cylinders with Geometric and Loading Imperfections," International Journal of Structural Stability and Dynamics, vol. 10, no. 4, pp. 623-644, 2010.

[20] M. A. Arbelo, R. Zimmermann, S. G. P. Castro, and R. Degenhardt, "Comparison of New Design Guidelines for Composite Cylindrical Shells Prone to Buckling," in Proceedings of the 9th International Conference on Composite Science and Technology (ICCST-9), Italy, 2013, pp. 96-111.

[21] B. Geier, H.-R. Meyer-Piening, and R. Zimmermann, "On the Influence of Laminate Stacking on Buckling of Composite Cylindrical Shells Subjected to Axial Compression," Composite Structures, vol. 55, no. 4, pp. 467-474, 2002.

[22] S. G. P. Castro, R. Zimmermann, M. A. Arbelo, and R. Degenhardt, "Exploring the Constancy of the Global Buckling Load After a Critical Geometric Imperfection Level in ThinWalled Cylindrical Shells for Less Conservative Knock-down Factors," Thin-Walled Structures, vol. 72, pp. 76-87, Nov. 2013.

[23] S. G. P. Castro, R. Zimmermann, M. A. Arbelo, R. Khakimova, M. W. Hilburger, and R. Degenhardt, "Geometric Imperfections and Lower-Bound Methods Used to Calculate Knockdown Factors for Axially Compressed Composite Cylindrical Shells," Thin-Walled Structures, vol. 74, pp. 118-132, Jan. 2014.

[24] W. T. Haynie, M. W. Hilburger, M. Bogge, and B. Kriegesmann, "Validation of LowerBound Estimates for Compression-Loaded Cylindrical Shells," in Proceedings 53rd AIAA/ASME/ASCE/AHS/ASC Structures, Structural Dynamics and Materials Conference, Honolulu, USA, 2012, p. AIAA Paper 2012-1689.

[25] P. Steinmüller, R. Degenhardt, and K. Rohwer, "Investigations of Axially Loaded Unstiffened CFRP Cylindrical Shells Subject to Single Perturbation Loads," presented at the 2nd International Conference on Buckling and Postbuckling Behaviour of Composite Laminated Shell Structures with COCOMAT Workshop, Braunschweig, Germany, 2008.

[26] L. Wullschleger and H.-R. Meyer-Piening, "Buckling of geometrically imperfect cylindrical shells - definition of a buckling load," International Journal of Non-Linear Mechanics, vol. 37, no. 4-5, pp. 645-657, Jun. 2002.

[27] R. C. Tennyson, "The Effects of Unreinforced Circular Cutouts on the Buckling of Circular Cylindrical Shells Under Axial Compression," J. Eng. Ind, vol. 90, no. 4, pp. 541-546, Nov. 1968.

[28] S. Toda, "Buckling of cylinders with cutouts under axial compression," Experimental Mechanics, vol. 23, no. 4, pp. 414-417, Dec. 1983.

[29] M. A. Arbelo, A. Herrmann, S. G. P. Castro, R. Khakimova, R. Zimmermann, and R. Degenhardt, "Investigation of Buckling Behavior of Composite Shell Structures with Cutouts," Appl Compos Mater, vol. 22, no. 6, pp. 623-636, Nov. 2014.

[30] B. Kriegesmann, M. W. Hilburger, and R. Rolfes, "The Effects of Geometric and Loading Imperfections on the Response and Lower-Bound Buckling Load of a Compression-Loaded Cylindrical Shell," in Proceedings 53rd AIAA/ASME/ASCE/AHS/ASC Structures, Structural Dynamics and Materials Conference, Honolulu, USA, 2012, p. AIAA Paper 2012-1864. 
[31] M. A. Arbelo, R. Degenhardt, S. G. P. Castro, and R. Zimmermann, "Numerical Characterization of Imperfection Sensitive Composite Structures," Composite Structures, vol. 108, pp. 295-303, Feb. 2014.

[32] R. Degenhardt, A. Kling, A. Bethge, J. Orf, L. Kärger, R. Zimmermann, K. Rohwer, and A. Calvi, "Investigations on Imperfection Sensitivity and Deduction of Improved Knock-down Factors for Unstiffened CFRP Cylindrical Shells," Composite Structures, vol. 92, no. 8, pp. 1939-1946, 2010.

[33] C. Hühne, "Robuster Entwurf beulgefährdeter, unversteifter Kreiszylinderschalen aus Faserverbundwerkstoff (Ph. D. thesis)," Technische Universität Carolo-Wilhelmina zu Braunschweig, 2008.

[34] R. Wagner and C. Hühne, "A New Design Concept for Cylindrical Composite Shells Under Axial Compression," in 16th European Conference on Composite Materials, Seville, Spain, 2014.

[35] W. A. Waters, "Effects Initial Geometric Imperfections on the Behavior of GraphiteEpoxy Cylinders Loaded in Compression," Old Dominion University, Norfolk, Virginia, USA, Master thesis, 1996.

[36] R. C. Tennyson and D. B. Muggeridge, "Buckling of Laminated Anisotropic Imperfect Circular Cylinders under Axial Compression," Journal of Spacecraft, vol. 10, no. 2, pp. 143-148, 1973.

[37] R. M. Jones, Mechanics Of Composite Materials. CRC Press, 1998.

[38] J. Arbocz and J. M. A. M. Hol, "Koiter's Stability Theory in a Computeraided Engineering (CAE) Environment," International Journal of Solids and Structures, vol. 26, no. 910, pp. 945-973, 1990.

[39] G. A. Cohen, "Effect of a Nonlinear Prebuckling State on the Postbuckling Behaviorand Imperfect on Sensitivity of Elastic Structures," AIAA Journal, vol. 6, no. 8, pp. 1616-1619, 1968. 